\title{
FATORES CLIMÁTICOS NA DINÂMICA POPULACIONAL DE Anastrepha spp. (DIPTERA: TEPHRITIDAE) E DE Scymnus spp. (COLEOPTERA: COCCINELLIDAE) EM UM POMAR EXPERIMENTAL DE GOIABA (Psidium guajava L. $)^{1}$
}

\author{
RICARDO APARECIDO CALORE ${ }^{2}$, JULIO CÉSAR GALLI ${ }^{3}$, WILSON CARLOS PAZINI ${ }^{4}$, \\ ROGÉRIO TEIXEIRA DUARTE ${ }^{5}$, JULIANA ALTAFIN GALLI ${ }^{6}$
}

RESUMO-Este trabalho objetivou caracterizar a dinâmica populacional de Anastrepha spp. e de Scymnus spp. em pomar experimental semiorgânico de goiaba (Psidium guajava L.), em Pindorama-SP, na Agência Paulista de Tecnologia dos Agronegócios (APTA) e correlacioná-la com fatores meteorológicos. Para o levantamento da dinâmica populacional, os espécimes foram monitorados com armadilhas adesivas amarelas ( $25 \mathrm{~cm} \times 9,5 \mathrm{~cm})$, trocadas a cada 15 dias, no período de um ano (entre junho de 2009 e junho de 2010). Os insetos foram avaliados e quantificados no Laboratório de Seletividade Ecológica da UNESP-FCAV em Jaboticabal-SP. Observou-se a ocorrência de Anastrepha spp. e Scymnus spp. durante todo o período de amostragem. Com base nos resultados obtidos e nas condições de desenvolvimento do presente trabalho, foram possíveis as seguintes conclusões: a) Ocorre aumento na densidade populacional de Anastrepha spp. com o aumento das temperaturas mínima, média e máxima; b) Os picos populacionais de Anastrepha spp. ocorrem de janeiro a março e coincidem com o período de disponibilidade de frutos maduros no pomar de goiaba; c) Constatam-se as maiores ocorrências do predador Scymnus spp. no período de setembro a dezembro, e as menores ocorrências, em fevereiro e março; d) As precipitações não interferem na dinâmica populacional de Anastrepha spp. e de Scymnus spp..

Termos para indexação: Insecta, Manejo integrado de pragas, Fatores meteorológicos.

\section{CLIMATIC FACTORS IN POPULATION DYNAMIC OF Anastrepha spp. (DIPTERA: TEPHRITIDAE) AND Scymnus spp. (COLEOPTERA: COCCINELLIDAE) IN AN EXPERIMENTAL ORCHARD OF GUAVA (Psidium guajava L.)}

\begin{abstract}
The objective of this study was to evaluate the population dynamic of Scymnus spp. and Anastrepha spp. and their correlations between meteorological factors in an experimental semi-organic guava (Psidium guajava L.) orchard in the experimental field of APTA (Agência Paulista de Tecnologia dos Agronegócios) in Pindorama, SP. For the survey of population dynamic, the specimens were collected with yellow sticky traps $(25 \mathrm{~cm} \times 9.5 \mathrm{~cm})$ which were replaced every 15 days in a period of one year (between June 2009 and June 2010). It was observed the occurrence of Scymnus spp. and Anastrepha spp. throughout the sampling period. The insects were assessed and quantified at the Laboratory of Ecological Selectivity at FCAV-UNESP in Jaboticabal-SP. Based on the results obtained and the conditions of development of this study, it was possible to conclude: a) There is an increase in population of Anastrepha spp. with the increase of minimum, average and maximum temperatures; b) The population peak of Anastrepha spp. occur from January to March and coincides with the period of availability of ripe fruits in the guava orchard; c) The highest occurrences of predator Scymnus spp. were from September to December and the lowest rates in February and March d) The rainfall did not interfere in the population dynamic of Scymnus spp. and Anastrepha spp. Index terms: Insecta, Integrated Pest Management, meteorological factors.
\end{abstract}

\footnotetext{
(Trabalho 145-12). Recebido em: 15-05-2012. Aceito pra publicação em: 07-01-2013.

${ }^{2}$ Eng. Agr. Mestre em Entomologia Agrícola, UNESP/FCAV, Jaboticabal - SP. E-mail: ricardocalore@yahoo.com.br ${ }^{3}$ Eng. Agr. Prof. Dr. em Entomologia Agrícola. - UNESP/FCAV, Jaboticabal - SP. E-mail: jcgalli@fcav.unesp.br ${ }^{4}$ Eng. Agr. Dr. em Entomologia Agrícola. -UNESP/FCAV, Jaboticabal - SP. E-mail: wpazini@fcav.unesp.br

${ }^{5}$ Eng. Agr. Mestre em Entomologia Agrícola - UNESP/FCAV, Jaboticabal - SP. E-mail: rogerioteixeira_1@hotmail.com

${ }^{6}$ Eng. Agr. Agência Paulista de Tecnologia dos Agronegócios, Apta Centro Norte, Cx Postal 24, CEP 15830-000, Pindorama - SP.

E-mail: julianagalli@apta.sp.gov.br
} 


\section{INTRODUÇÃO}

O aumento da competitividade da goiaba e seus derivados fazem- se necessários no Brasil, pela possibilidade de alavancar grande volume de exportação, proporcionando divisas importantes para o País, além de proporcionar a geração de milhares de empregos em toda a cadeia produtiva.

A utilização de alta tecnologia na produção de goiaba, aliada à aptidão na região de Jaboticabal-SP, em produzir frutas, favoreceu o desenvolvimento da cultura e da agroindústria. Assim, novas cultivares, irrigação e mudanças no sistema de podas dos pomares, visando a aumentar a produtividade e a fornecer frutas o ano todo, agravaram consideravelmente os danos diretos e indiretos ocasionados pelas pragas, com destaque para as moscas-das-frutas.

As larvas das moscas-das-frutas causam sérios prejuízos à fruticultura, pois alimentam-se da polpa dos frutos, tornando-os impróprios para o consumo in natura e para a industrialização. Em alguns frutos, como a maçã, a tentativa de oviposição das moscas-das-frutas já provoca deformação nos frutos, tornando-os sem valor comercial (ORTH et al., 1986). As moscas-das-frutas são de ocorrência endêmica na região de Jaboticabal-SP, fato que as tornam, constantemente, pragas em potencial na goiaba.

De acordo com Dória et al. (2004), o principal entrave à exportação desses frutos é a imposição de barreiras quarentenárias pelos países importadores, visando a impedir a introdução das mosca-dasfrutas Ceratitis capitata (Wiedemann 1824) (Dip.: Tephritidae) e Anastrepha spp. (Dip.: Tephritidae). Desse modo, essas pragas tornam-se importantes, não só pelos danos diretos causados pelas larvas, mas também por impedir a comercialização de várias espécies frutíferas no mercado externo, como goiaba, melão, manga, carambola, maçã, pêssego, ameixa (HICKEL;DUCROQUET, 1993; MACHADO et al., 1995; KOVALESKI et al., 1999; MALAVASI; ZUCCHI, 2000; GARCIA; CORSEUIL, 2004).

Devido à grande exigência dos países importadores com relação à ausência de pragas e resíduos químicos, junto à conscientização ambiental de consumidores e produtores brasileiros, o manejo das pragas da goiabeira tem-se aperfeiçoado. Assim, o monitoramento da flutuação populacional das pragas e dos inimigos naturais, utilizando-se de armadilhas, está sendo empregado com destaque. $\mathrm{O}$ ensacamento dos frutos, a liberação de machos estéreis e a utilização de parasitoides têm-se sobressaído no controle das pragas da goiabeira (UCHOA-FERNANDES; ZUCCHI, 1999; GARCIA et al., 2003).

A sazonalidade dos elementos meteorológicos pode influenciar direta ou indiretamente em alguns insetos da goiaba. Muitos elementos podem atuar diretamente na mortalidade ou afetar o desempenho dos insetos, através de alterações de oviposição, alimentação, crescimento, desenvolvimento e migração (HOPKINS; MEMMOTT, 2003). Indiretamente, os elementos meteorológicos podem afetar os insetos por influenciar na atividade dos inimigos naturais e alterar a qualidade dos recursos através de mudanças fisiológicas e bioquímicas na planta hospedeira (VARLEY et al., 1973; HOPKINS; MEMMOTT, 2003). Dentre os elementos meteorológicos, a temperatura, a umidade relativa, a precipitação pluviométrica e a velocidade do vento são os principais fatores relacionados à dinâmica populacional de insetos em diversos agroecossistemas (WALLNER, 1987). Contudo, a evaporação, a insolação e o fotoperíodo também podem ser importantes para algumas espécies (TAKEDA;SKOPIK, 1997).

Também a forma da condução da cultura pode influenciar na diversidade de pragas e de inimigos naturais, e deve ser objeto de estudos com o intuito de melhorar a compreensão das interações plantas, pragas e inimigos naturais relacionados com a implantação de um sistema de manejo com bases ecológicas, econômicas e seguras ao homem.

O controle químico e o controle biológico são táticas possíveis para a redução da população de pragas. Porém, o levantamento populacional é o primeiro passo para se chegar ao manejo integrado de praga, observando-se os picos populacionais e as relações com fatores abióticos (BARBOSA et al., 2001; MENEZES JR.; PASINI, 2001).

É de conhecimento técnico-científico que o desenvolvimento de Programas de Manejo Integrado de Pragas (MIP) depende grandemente de estudos básicos de dinâmica populacional e determinação da importância relativa das forças que regulam o crescimento populacional de insetos. Dessa forma, torna-se necessário que se desenvolvam pesquisas para se conhecerem quais são os fatores e como eles interferem na intensidade de ataque das pragas à cultura de goiaba.

Entre os inimigos naturais presentes na goiabeira, destacam-se as joaninhas da família Coccinellidae, que são importantes predadores por apresentarem grande diversidade alimentar. A maioria das espécies pertencentes a essa família é entomófaga, alimentando-se de espécies de hemípteros, ácaros e larvas de coleópteros desfolhadores (CLAUSEN, 1972). Segundo 
Olkowsky et al. (1990), os coccinelídeos estão entre os mais conhecidos predadores de insetos e ocorrem na maioria das regiões do mundo, controlando pragas de inúmeras culturas.

A melhoria do conhecimento do agroecossistema da goiabeira, como uma unidade ecológica complexa, possibilita o aperfeiçoamento de programas de MIP que são importantes regionalmente.

O objetivo deste trabalho foi caracterizar a dinâmica populacional de Anastrepha spp. e de Scymnus spp. (Col.: Coccinellidae) em pomar experimental semiorgânico de goiaba na região de Pindorama-SP, e correlacioná-la com fatores meteorológicos.

\section{MATERIAL E MÉTODOS}

$\mathrm{O}$ experimento foi instalado em um pomar experimental semiorgânico de goiaba (o qual possui todas as características de um pomar orgânico, mas sem a certificação oficial) na região de PindoramaSP, em campo experimental da Agência Paulista de Tecnologia dos Agronegócios (APTA). O pomar apresentava uma coleção de 92 genótipos de goiabeira, com 14 anos de idade, com porte uniforme e plantação em nível. O cultivo foi conduzido sem regime de poda, sem irrigação, não recebeu adubação química e nenhum tipo de pulverização de produtos fitossanitários. O controle das plantas invasoras foi feito com uma ceifadeira acoplada ao trator.

Foram instaladas três armadilhas adesivas amarelas (Biocontrole ${ }^{\circledR}$ dupla face $25 \mathrm{~cm} \times 9,5$ $\mathrm{cm})$ em cada um dos genótipos: Rubi Supreme (Vermelha), IAC-4 Vermelha, Rica-J-2-UNESP, Paluma UNESP (vermelha), Saito (Vermelha), Supreme e Kioshi 3 (vermelha), totalizando 21 armadilhas, no período de junho de 2009 a junho de 2010. Estas foram posicionadas acerca de dois metros do solo, na parte externa da planta, quadrante sul da copa da goiabeira e trocadas a cada 15 dias, continuamente.

Cada armadilha retirada do campo foi acondicionada em uma pasta de plástico e conduzida ao Laboratório de Seletividade Ecológica do Departamento de Fitossanidade da UNESP-FCAV, em Jaboticabal-SP, onde foram feitas as avaliações. Os insetos foram identificados, quantificados e registrados em ficha de laboratório na data de amostragem. Os dados meteorológicos foram fornecidos pelo posto meteorológicos da fazenda experimental da APTA - Pindorama-SP, localizado acerca de 800 metros do local do experimento.

Com os dados obtidos, foram calculados as correlações lineares simples entre as moscas- das-frutas do gênero Anastrepha spp. e os fatores meteorológicos: temperatura $\left({ }^{\circ} \mathrm{C}\right)$ (mínima, média e máxima) e precipitação pluviométrica $(\mathrm{mm})$.Também foram verificadas as correlações lineares simples entre os fatores meteorológicos anteriormente descritos e o predador Scymnus spp..

\section{RESULTADOS E DISCUSSÃO}

As maiores densidades populacionais de moscas do gênero Anastrepha spp. ocorreram de janeiro a março/2010, sendo que o pico populacional da praga ocorreu em 15-02-2010, com um total de 2.440 moscas, seguido pelas coletas realizadas em $1^{\circ}-03-2010$ e $15-03-2010$, com um total de 1.537 e 1.559 moscas, respectivamente, coletadas nas 21 armadilhas adesivas amarelas (Figura 1). Constatou-se que as maiores incidências de moscasdas-frutas do gênero Anastrepha coincidiu com a época de frutificação do pomar de goiaba em Pindorama. Chiardia et al. (2004), em um trabalho similar, observaram que os picos de captura de moscas do gênero Anastrepha ocorreram de março a junho. Santos et al. (1998) observaram que o pico populacional de moscas do gênero Anastrepha, no município de Mossoró-RN, ocorreu em junho, portanto em época diferente da relatada nesta pesquisa. Araújo e Zucchi (2003), ao estudarem os níveis de infestação das espécies de Anastrepha spp. em goiaba, verificaram que os maiores níveis de infestação e picos populacionais ocorreram de maio a julho. Os autores relatam que, nas regiões semiáridas, a precipitação, juntamente com a disponibilidade de hospedeiros são os fatores preponderantes nos picos populacionais e não somente a disponibilidade de frutos, como os resultados obtidos neste trabalho.

Constatou-se uma densidade populacional de moscas do gênero Anastrepha relativamente alta, de outubro a novembro de 2009 , com destaque para a amostragem realizada em 26-10-2009, com um total de 518 moscas coletadas nas 21 armadilhas adesivas amarelas (Figura 1). Cabe inferir que havia próximo à localidade do pomar experimental outras culturas, como manga, citros e café, que podem ter servido de hospedeiros alternativos para as espécies de Anastrepha spp. e, com isso, influenciado na sua população, através da migração. Esta pesquisa não tinha como meta verificar a infestação de moscasdas-frutas nos diferentes genótipos. Entretanto, não foi constatado nenhuma variação na captura de Anastrepha spp. entre os sete genótipos de goiabeira durante o experimento.

As menores densidades populacionais de Anastrepha spp. ocorreram no período de abril 
a junho de 2009 (Figura 1). Foi constatado que não havia a presença de frutos no pomar, nesse período. Estes resultados corroboram os de Teles e Silva (2005), que relataram que a disponibilidade de frutos hospedeiros é o fator mais importante na determinação da ocorrência e da flutuação populacional de moscas-das-frutas e não fatores abióticos. Estes autores relataram, ainda, que a época de maior precipitação coincide com o período de maior frutificação e que, provavelmente, fatores não avaliados, como a disponibilidade de hospedeiros alternativos e sucessão hospedeira, exerceram influência na população de moscas.

As moscas-das-frutas do gênero Anastrepha resultaram no total de 8.832 indivíduos coletados nas 21 armadilhas adesivas amarelas, em todo o período de amostragem, sendo este o gênero de moscas-das-frutas predominante. Diversos outros pesquisadores, em trabalhos realizados no Brasil, também observaram que as moscas-das-frutas Anastrepha foi o gênero predominante (VELOSO, 1997; GARCIA; CORSEUIL, 1998; URAMOTO et al., 2003). Aluja et al. (1996) sugeriram que este tipo de comportamento seja considerado normal, dado que, embora diversas espécies de tefritídeos estejam presentes em um pomar, apenas uma ou duas espécies representam mais de $90 \%$ de todas as moscas-dasfrutas coletadas em armadilhas.

As correlações entre os fatores meteorológicos e a flutuação populacional das moscas-das-frutas apresentaram significância para as temperaturas mínima, média e máxima, indicando que o crescimento populacional da praga é favorecido em temperaturas mais elevadas (Tabela 1). Não houve correlação para a precipitação.

Dentre o complexo de inimigos naturais coletados nas armadilhas durante a realização do experimento, destacaram-se os espécimes Scymnus spp., Azya luteipes, Polybia spp. e Cycloneda sanguinea e das famílias Formicidae, Chrysopidae e Aracnidae (Tabela 2). Quantitativamente, Scymnus spp foi o espécime coletado em maior número. Este gênero, pertencente à família Coccinellidae, é predador importante e ocorrem na maioria das regiões do mundo (CLAUSEN, 1972; OLKOWSKY et al.,1990). Parajulle e Slosser (2003) realizaram um experimento com algodão, no Estado do Texas, para monitorar pragas e inimigos naturais com armadilhas adesivas amarelas, e também observaram que os espécimes mais encontrados foram Scymnus spp. No Nordeste brasileiro, Barbosa et al. (1999) realizaram pesquisa de eficiência e seletividade de inseticidas, em pomar de goiaba, e também verificaram que Scymnus spp. e espécimes de sirfídeos foram os inimigos naturais que apareceram em maior número.

O maior pico populacional de Scymnus spp. ocorreu em 29-06-2009, com um total de 705 indivíduos coletados nas 21 armadilhas adesivas amarelas e manteve-se num número relativamente alto em todo o período de amostragem, destacando-se no período de setembro a dezembro de 2009. Porém, apresentou menor ocorrência nos meses de fevereiro e março de 2010 (Figura 2). Constata-se que a maior incidência de Scymnus spp ocorre no período de baixa infestação de Anastrepha spp., indicando ser este inimigo natural mais eficiente na predação de outros insetos, como pulgões, cochonilhas, entre outros, e menos eficiente em relação às moscas-das-frutas. O refúgio destes insetos é dependente do tipo de planta, invasora estabelecida na região, talvez por características peculiares da própria planta, ou por estas abrigarem outros insetos pelos quais são fonte de alimento (BARBOSA et al. 2003). As densidades populacionais de Scymnus spp. apresentaram correlação linear significativa negativa, ou seja, inversamente proporcional para as temperaturas mínima, média e máxima (Figura 2) (Tabela 3 ).

Dentre os inimigos naturais coletados durante o experimento, alguns são agentes de controle potenciais de Anastrepha spp., como os parasitoides da família Braconidae (MARINHO et al., 2009; BITTENCOURT et al., 2011). Muito pouco se sabe sobre a ação dos predadores das moscas-das-frutas no Brasil, pela falta de relatos na literatura referente ao assunto, sendo, portanto, motivo de estudos para novas pesquisas. 
TABELA 1- Coeficiente de correlação linear simples calculado entre Anastrepha spp. e os fatores meteorológicos: temperaturas mínima (Tmín), média (Tméd) e máxima (Tmáx) $\left({ }^{\circ} \mathrm{C}\right)$, e precipitação acumulada por amostragem (PREC.) (mm) no período de junho de 2009 a junho de 2010. Pindorama-SP, 2009-2010.

* Significativo a $5 \%$ de probabilidade.

\begin{tabular}{cccc}
\multicolumn{4}{c}{ Coeficiente de correlação linear } \\
\hline Tmín $\left({ }^{\circ} \mathrm{C}\right)$ & Tméd $\left({ }^{\circ} \mathrm{C}\right)$ & Tmáx $\left({ }^{\circ} \mathrm{C}\right)$ & PREC. $(\mathrm{mm})$ \\
\hline $0,4501^{*}$ & $0,4790^{*}$ & $0,4822^{*}$ & $0,1013^{\text {ns }}$ \\
\hline
\end{tabular}

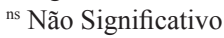

TABELA 2- Inimigos naturais adultos coletados em 21 armadilhas adesivas amarelas, em pomar de goiaba. Pindorama-SP, 2009-2010.

\begin{tabular}{|c|c|c|c|c|c|c|c|c|c|c|c|c|}
\hline \multirow{2}{*}{ Coletas } & \multicolumn{12}{|c|}{ Inimigos naturais } \\
\hline & $\mathbf{a}$ & b & c & d & $\bar{e}$ & f & $\mathbf{g}$ & $\mathbf{h}$ & $\overline{\mathbf{i}}$ & $\mathbf{j}$ & $\mathbf{k}$ & I \\
\hline $29-06-2009$ & 705 & 5 & 19 & 19 & 24 & 11 & 17 & 4 & 3 & 0 & 4 & 0 \\
\hline $13-07-2009$ & 353 & 4 & 26 & 24 & 25 & 11 & 46 & 0 & 5 & 10 & 0 & 0 \\
\hline $27-07-2009$ & 269 & 2 & 25 & 26 & 33 & 16 & 30 & 0 & 0 & 10 & 5 & 0 \\
\hline $17-08-2009$ & 199 & 3 & 17 & 3 & 19 & 10 & 8 & 0 & 0 & 2 & 0 & 0 \\
\hline $31-08-2009$ & 94 & 0 & 9 & 11 & 52 & 10 & 47 & 0 & 0 & 6 & 4 & 0 \\
\hline 14-09-2009 & 142 & 17 & 80 & 9 & 55 & 12 & 12 & 0 & 0 & 6 & 0 & 0 \\
\hline 28-09-2009 & 194 & 8 & 27 & 6 & 19 & 14 & 25 & 1 & 1 & 8 & 0 & 0 \\
\hline $13-10-2009$ & 429 & 114 & 100 & 13 & 20 & 13 & 23 & 1 & 0 & 0 & 4 & 2 \\
\hline $26-10-2009$ & 413 & 77 & 97 & 13 & 59 & 19 & 70 & 2 & 0 & 12 & 3 & 5 \\
\hline 09-11-2009 & 229 & 44 & 129 & 14 & 27 & 55 & 65 & 0 & 0 & 4 & 0 & 1 \\
\hline $21-11-2009$ & 262 & 0 & 103 & 10 & 14 & 22 & 51 & 8 & 0 & 0 & 0 & 3 \\
\hline $07-12-2009$ & 236 & 2 & 61 & 14 & 12 & 9 & 56 & 2 & 0 & 1 & 0 & 0 \\
\hline $21-12-2009$ & 164 & 0 & 94 & 18 & 21 & 11 & 98 & 0 & 1 & 1 & 0 & 0 \\
\hline $04-01-2010$ & 67 & 0 & 35 & 10 & 2 & 10 & 55 & 0 & 0 & 1 & 0 & 0 \\
\hline $18-01-2010$ & 101 & 0 & 58 & 11 & 3 & 26 & 34 & 0 & 0 & 0 & 0 & 1 \\
\hline $1^{\circ}-02-2010$ & 66 & 1 & 12 & 6 & 3 & 8 & 42 & 0 & 0 & 0 & 0 & 2 \\
\hline $15-02-2010$ & 51 & 0 & 9 & 3 & 6 & 7 & 27 & 0 & 0 & 0 & 0 & 2 \\
\hline $1^{\circ}-03-2010$ & 75 & 1 & 16 & 3 & 12 & 2 & 112 & 1 & 0 & 0 & 0 & 4 \\
\hline $15-03-2010$ & 87 & 0 & 22 & 7 & 9 & 6 & 18 & 0 & 0 & 0 & 0 & 1 \\
\hline $29-03-2010$ & 128 & 0 & 19 & 14 & 19 & 16 & 17 & 4 & 0 & 0 & 1 & 2 \\
\hline $12-04-2010$ & 193 & 2 & 21 & 6 & 17 & 13 & 19 & 1 & 0 & 0 & 0 & 2 \\
\hline $26-04-2010$ & 202 & 1 & 26 & 6 & 20 & 20 & 32 & 1 & 0 & 0 & 0 & 2 \\
\hline $10-05-2010$ & 263 & 0 & 9 & 10 & 22 & 25 & 27 & 2 & 0 & 0 & 0 & 8 \\
\hline $24-05-2010$ & 297 & 1 & 10 & 8 & 27 & 15 & 21 & 3 & 0 & 0 & 0 & 1 \\
\hline $07-06-2010$ & 128 & 0 & 6 & 7 & 8 & 9 & 5 & 2 & 0 & 0 & 0 & 0 \\
\hline $21-06-2010$ & 264 & 0 & 7 & 6 & 14 & 20 & 25 & 0 & 0 & 0 & 1 & 0 \\
\hline Total & 5611 & 282 & 1037 & 277 & 542 & 390 & 982 & 32 & 10 & 61 & 22 & 36 \\
\hline
\end{tabular}

TABELA3 - Coeficiente de correlação linear simples calculado entre Scymnus spp. e os fatores meteorológicos: temperaturas mínima (Tmín), média (Tméd) e máxima (Tmáx) $\left({ }^{\circ} \mathrm{C}\right)$, e precipitação acumulada (Prec) (mm) no período de junho de 2009 a junho de 2010. Pindorama-SP, 2009-2010.

\begin{tabular}{lcccc}
\hline & \multicolumn{4}{c}{ Coeficiente de correlação linear } \\
\cline { 2 - 5 } Scymmus spp. & $-0,5207^{* *}$ & $-0,5160^{* *}$ & $-0,4601^{*}$ & $0,2759^{\text {ns }}$ \\
\cline { 2 - 5 }
\end{tabular}

\footnotetext{
** Significativo a $1 \%$ de probabilidade.

* Significativo a 5\% de probabilidade.

${ }^{n s}$ Não Significativo
} 


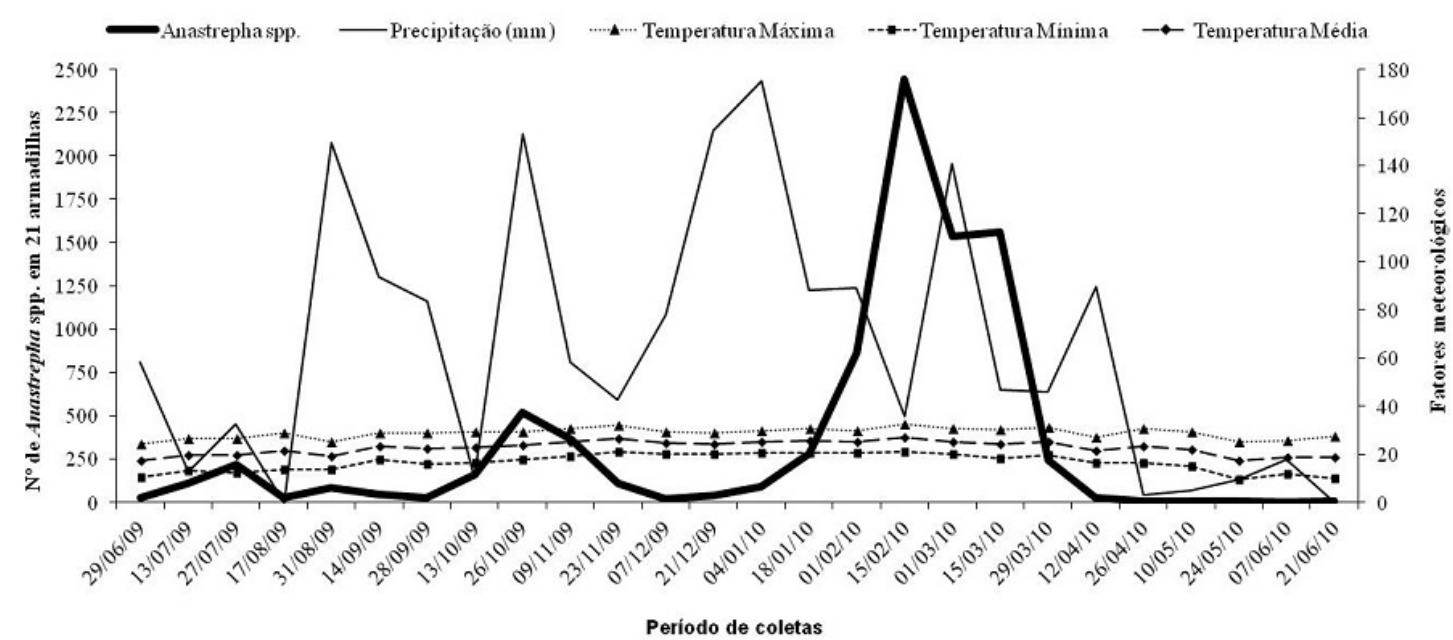

FIGURA 1 - Número de Anastrepha spp. capturadas em 21 armadilhas adesivas amarelas, e ocorrência de fatores meteorológicos em pomar de goiaba, no período de junho de 2009 a junho de 2010. Pindorama-SP, 2009-2010.

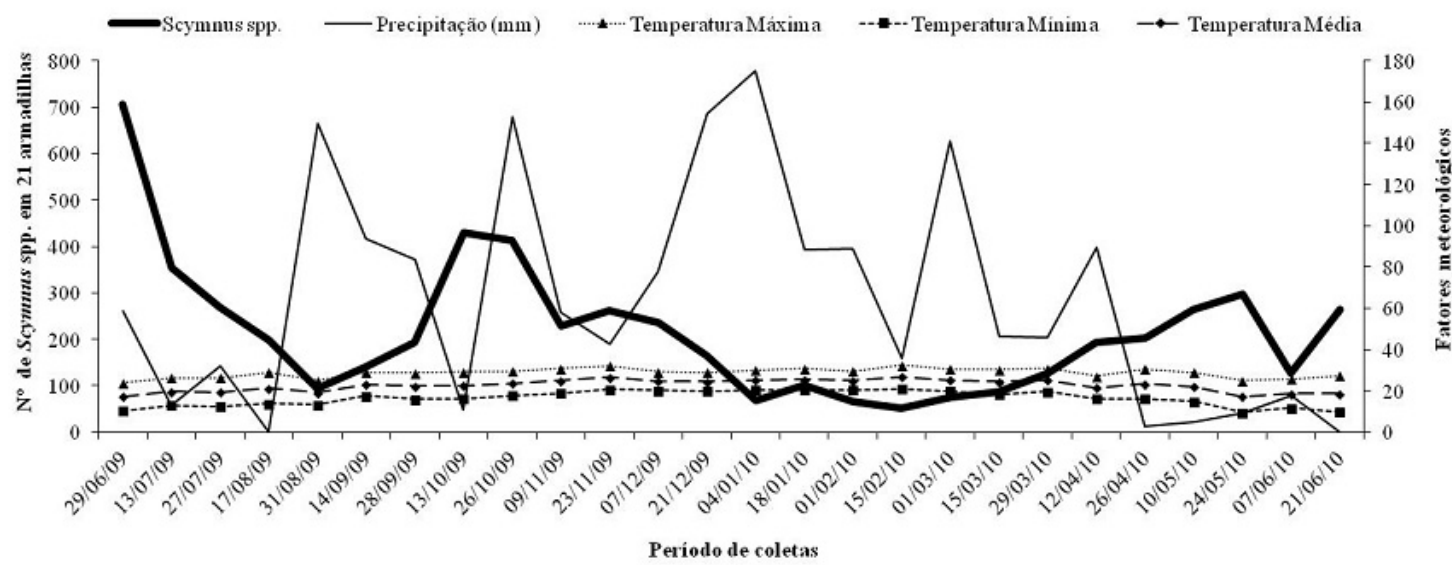

FIGURA 2 - Número total de Scymnus spp. capturados em 21 armadilhas adesivas amarelas, e ocorrência de fatores meteorológicos, em pomar de goiaba, no período de junho de 2009 a junho de 2010. Pindorama-SP, 2009-2010.

\section{CONCLUSÕES}

Com base nos resultados obtidos e nas condições de desenvolvimento do presente trabalho, foi possível tirar as seguintes conclusões:

a) Ocorre aumento na densidade populacional de Anastrepha spp. com o aumento das temperaturas mínima, média e máxima.

b) Os picos populacionais de Anastrepha spp. ocorrem de janeiro a março e coincidem com o período de disponibilidade de frutos maduros no pomar de goiaba. c) As maiores populações Scymnus spp. ocorrem no período de setembro a dezembro, e as menores, nos meses de fevereiro e março.

d) As precipitações não interferem nas dinâmicas populacionais de Scymnus spp. e de Anastrepha spp..

\section{AGRADECIMENTOS}

Os autores agradecem à chefia da Estação Experimental da Agência Paulista de Tecnologia dos Agronegócios-APTA, Centro Norte, em PindoramaSP, na pessoa do Dr. Antônio Lúcio Melo Martins, por ceder a área experimental. 


\section{REFERÊNCIAS}

ALUJA, M.; CELEDONIO-HURTADO, H.; LIEDO, P.; CABRERA, M.; CASTILLO, F.; GUILLÉN, J.; RIOS, E. Seasonal population fluctuations and ecological implications for management of Anastrepha fruit flies (Diptera: Tephritidae) in commercial orchards in Southern Mexico. Journal of Economic Entomology, Lanham, v. 89, n. 3, p. 654-667, 1996.

ARAÚJO, E. L; ZUCCHI, R. A. Moscas-das-frutas (Diptera: Tephritidae) em goiaba (Pisidium guajava L.) em Mossoró, RN. Arquivos do Instituto Biológico, São Paulo, v. 70, n. 4, p. 459-465, 2003.

BARBOSA, F. R.; FERREIRA, R. G.; KIILL, L. H. P.; DE SOUZA, E. A.; MOREIRA, W. A.; DEALENCAR, J. A.; HAJI, F. N. P. Nível de dano, plantas invasora hospedeiras, inimigos naturais e controle do psilídeo da goiabeira (Triozoida sp.) no Submédio São Francisco. Revista Brasileira de Fruticultura, Jaboticabal, v. 25, n. 3, p. 425-428, 2003.

BARBOSA, F.R.; SANTOS, A.P. dos; HAJI, A.T.; MOREIRA, W.A.; HAJI, F.N.P.; ALENCAR, J.A. Eficiência e seletividade do Imidacloprid e lambdacyhalothrin no controle do psilídeo (Triozoida sp.), em goiabeira. Revista Brasileira de Fruticultura, Jaboticabal, v. 21, n. 3, p. 385- 387, 1999.

BARBOSA, F.R.; SANTOS, A.P.; MOREIRA, W.A.; LIMA, J.A.S.; ALENCAR, J.A.; HAJI, F.N.P. Eficiência e seletividade de inseticidas no controle do psilídeo (Triozoida, sp.) em goiabeira. Pesticidas: Revista de Ecotoxicologia e Meio Ambiente, Curitiba, v.11, p. 45-52, 2001.

BITTENCOURT, M.A.L.; SILVA, A.C.M.; SILVA, V.E.S.; BONFIM, Z.F.; GUIMARÃES, J.A.; SOUZA FILHO, M.F.; ARAÚJO, E.L. Moscas-das-frutas (Diptera: Tephritidae) e seus parasitoides (Hymenoptera: Braconidae) associados às plantas hospedeiras no sul da Bahia. Neotropical. Entomology, Londrina, v.40, n.3, p. 405-406, 2011.

CHIARDIA, L. A.; MILANEZ, J. M.; DITRICHI,R. Flutuação populacional de moscas-das-frutas em pomares de cítrus no oeste de Santa Catarina, Brasil. Ciência Rural, Santa Maria, v. 34, n. 2, 2004.

CLAUSEN, C P. Entomophagous insects. London: Hofner Publishing Company, 1972. 688p.
DÓRIA, H. O. S.; BORTOLI, S. A. de; ALBERGARIA, N. M. M. S. de. Influência do tratamento térmico na eliminação de Ceratitis capitata em frutos de goiaba (Psidium guajava L.). Acta Scientiarum. Animal Science, Maringá, v. 26, n. 1, p. 107-111, 2004.

GARCIA, F. R. M.; CAMPOS, J. V.; CORSEUIL, E. Flutuação populacional de Anastrepha fraterculus (Wiedemann, 1830) (Diptera, Tephritidae) na região oeste de Santa Catarina, Brasil. Revista Brasileira de Entomologia, Curitiba, v.47, n.3, 2003.

GARCIA, F. R. M.; CORSEUIL, E. Flutuação populacional de Anastrepha fraterculus (Wiedemann) e Ceratitis capitata (Wiedemann) (Diptera, Tephritidae) em pomares de pessegueiro em Porto Alegre, Rio Grande do Sul. Revista Brasileira de Zoologia, Curitiba, v. 15, n. 1, p. 153-158, 1998.

GARCIA, F. R. M.; CORSEUIL, E. Native hymenopteran parasitoids associated with fruit flies (Diptera: Tephritidae) in Santa Catarina State, Brazil. Florida Entomologist, Gainesville, v. 87, n. 4, p. 517-521, 2004.

HICKEL, E. R.; DUCROQUET, J. P. H. Flutuação populacional de espécies de Anastrepha sp. (Diptera: Tephritidae) relacionadas com a fenologia de frutificação do pêssego e ameixa em Santa Catarina. Anais da Sociedade Entomológica do Brasil, Jaboticabal, v. 22, p. 591-596, 1993.

HOPKINS, G. W.; MEMMOTT, J. Seasonality of a tropical leaf-mining moth: leaf availability versus enemy-free space. Ecological Entomology, Hoboken, v.28. n.6, p. 687-693, 2003.

KOVALESKI, A.; URAMOTO, K.; SUGAYAMA, R. L.; CANAL, D. N. A.; MALAVASI, A. A survey of Anastrepha Schiner (Diptera: Tephritidae) species in the apple growing área of the state of Rio Grande do Sul, Brasil. Revista Brasileira de Entomologia, Curitiba, v. 43, p. 229-234, 1999.

MACHADO, A. E.; SALLES, L. A. B.; LOECK, A. E. Exigências térmicas de Anastrepha fraterculus (Wied.) e estimativa do número de gerações em Pelotas-RS. Anais da Sociedade Entomológica do Brasil, Jaboticabal, v. 24, p. 573-579, 1995.

MALAVASI, A.; ZUCCHI, R. A. Moscas-das-frutas de importância econômica no Brasil. São Paulo: Holos, p.327, 2000. 
MARINHO, C. F.; SOUZA-FILHO, M. F.; RAGA, A.; ZUCCHI, R. A. Parasitoides (Hymenoptera: Braconidae) de moscas-das-frutas (Diptera: Tephritidae) no Estado de São Paulo: plantas associadas e parasitismo. Neotroprical. Entomoolgy, Londrina, v.38, n.3, p. 321-326, 2009.

MENEZES Jr., A.M.; PASINI, A. Parasitoides (Hymenoptera: Chalcidóidea) Associados à Triozoida limbata (Enderlein) (Hemíptera: Psyllidae) sobre a goiabeira, Psidium guajava L. (Myrtaceae) na região norte do Paraná. In: SIMPÓSIO DE CONTROLE BIOLÓGICO, 7., 2001, Poços de Caldas. Anais... p. 344.

OLKOWSKY, W.; ZHANG, A.;TERS, P. Improved biocontrol techiniques with lady beetles. The IPM Practioner Monitoring the Field of Pest Management, v.12, p. 1-12, 1990.

ORTH, A.; RIBEIRO, L.G.; REIS FILHO, W. Manejo de pragas. In: EMPRESA CATARINENSE DE PESQUISAAGROPECUÁRIA. Manual da cultura da macieira. Florianópolis, 1986. p. 341-379.

PARAJULLE, M. N.; SLOSSER, J.E. Potential of yellow sticky traps for lady beetle survey in cotton. Journal of Economic Entomology, Lanham, v. 96, n.1, p. 239-245, 2003.

SANTOS, J. D.; SANTOS, J. J.R.; CARDOSO, E. A.; SOUZA, R.P.; FILGUEIRA, M. A.; CHAVES, J. W.N. Flutuação populacional de moscas-das-frutas do gênero Anastrepha (Diptera-Tephritidae) em goiabeira (Psidium guajava 1.) no município de Mossoró-RN-Brasil. Caatinga, Mossoró, v.11, n. 1 p.91-93, 1998.
TAKEDA, M.; SKOPIK, S. D.; Photoperiodic time measurement and related physiological meckanisms in insects an mites. Annual Review of Entomology, Palo Alto, v.42, p.323-349, 1997.

TELES, B. R.; SILVA, N. M. Flutuação Populacional de Espécies de Anastrepha Schiner (Diptera: Tephritidae) na região de Manaus-AM. Neotropical Entomology, Londrina, v.5, n.34, p.733-741, 2005.

UCHOA-FERNANDES, M. A.; ZUCCHI, R. A. Metodología de colecta de tephritidae y lonchaeidae frugívoros (Díptera: Tephritoidea) y sus parasitoides (Hymenoptera). Anais da Sociedade Entomológica do Brasil, Jaboticabal, v. 28, p. 601-610, 1999.

URAMOTO, K.; WALDER, J. M. M.; ZUCCHI, R. A. Flutuação populacional de moscas-das-frutas do gênero Anastrepha Schiner, 1868 (Diptera, Tephritidae) no Câmpus "Luiz de Queiroz”, Piracicaba, São Paulo. Arquivos do Instituto Biologico, São Paulo, v. 70, n. 4, p. 459-465, 2003.

VARLEY, G.C.; GRADWELL, G.R.; HASSEL, M.P. Insect population ecology. Oxford: Blackwell Scientific Publications, 1973. 212p.

VELOSO, V.R.S. Dinâmica populacional de Anastrepha spp. E Ceratitis capitata (Wied., 1824) (Diptera, Tephritidae) nos cerrados de Goiás. 1997. 115 f. Tese (Doutorado) - Universidade Federal de Goiânia, Goiânia, 1997.

WALLNER, W.E. Factors affecting insect population dynamics: differences between outbreack an nonoutbreack species. Annual Review of Entomology, Palo Alto, v.32, p.317-340, 1987. 